PROCEEDINGS OF THE

AMERICAN MATHEMATICAL SOCIETY

Volume 140, Number 12, December 2012, Pages 4337-4350

S 0002-9939(2012)11278-X

Article electronically published on April 11, 2012

\title{
CONES AND CONVEX BODIES WITH MODULAR FACE LATTICES
}

DANIEL LABARDINI-FRAGOSO, MAX NEUMANN-COTO, AND MARTHA TAKANE

(Communicated by Jim Haglund)

Dedicated to Claus M. Ringel on the occasion of his 65th birthday

AbStRaCt. If a convex body $C$ in $\mathbb{R}^{n}$ has modular and irreducible face lattice and $C$ is not strictly convex, there is a face-preserving homeomorphism from $C$ to a set of positive-semidefinite Hermitian matrices of trace 1 over $\mathbb{R}, \mathbb{C}$ or $\mathbb{H}$, or $C$ has dimension 8,14 or 26 .

\section{INTRODUCTION}

Let $C$ be a convex body in $\mathbb{R}^{n}$. A subset $F$ of $C$ is a face of $C$ if every open interval in $C$ that contains a point of $F$ is contained in $F$. An extreme point is a 1-point face. If $S$ is any subset of $C$, the face generated by $S$ is the minimal face of $C$ containing $S$. The set $\mathcal{F}(C)$ of all faces of $C$ ordered by inclusion is a lattice, which is always atomic, algebraic and complemented, as each face is generated by a finite number of extreme points and for every face $F$ there exists a face $G$ such that $F \wedge G=\emptyset$ and $F \vee G=C$. We want to consider convex bodies for which the lattice $\mathcal{F}(C)$ is modular. An atomic lattice is modular if and only if it is ranked (the lengths of all maximal chains between 2 elements are the same) and the rank (the length of a chain between 0 and $x)$ satisfies $r k(x)+r k(y)=r k(x \vee y)+r k(x \wedge y)$ for all $x$ and $y$ [4. The only examples of convex bodies with modular face lattice and a finite number of faces are the simplices [2, but beautiful examples with an infinite number of faces are given by the sets of positive-semidefinite Hermitian matrices of trace 1 over $\mathbb{R}, \mathbb{C}$ and $\mathbb{H}[3]$. The main purpose of this paper is to show that in dimensions other than 8,14 and 26, all convex bodies with modular and irreducible face lattices are deformations of these examples, in the sense that there is a face-preserving homeomorphism between them. The natural correspondence between convex bodies in $\mathbb{R}^{n}$ and closed cones in $\mathbb{R}^{n+1}$ gives analogous results for cones.

The paper is organized as follows. In section 2 we show that each convex body $C$ with modular face lattice is a convex join of convex bodies whose face lattices are modular and irreducible. It is known that modular and irreducible lattices correspond to abstract projective spaces. We review their basic properties and describe the examples of convex bodies whose face lattices determine the classical projective spaces over $\mathbb{R}, \mathbb{C}, \mathbb{H}$ and an octonionic projective plane.

Received by the editors February 20, 2009 and, in revised form, May 24, 2011.

2010 Mathematics Subject Classification. Primary 52A20, 06C05, 51A05, 15B48.

Key words and phrases. Convex, face lattice, modular, Hermitian matrix, projective space.

Research partially supported by PAPIIT grants IN103508, IN101309 and a PASPA fellowship.

(C)2012 American Mathematical Society

Reverts to public domain 28 years from publication 4337 
In section 3 we prove that if the face lattice of a convex body $C$ determines a projective space, it must be a topological projective space that is compact and connected. This is used to prove that, in most cases, the set of extreme points of $C$ determines a classical projective space over $\mathbb{R}, \mathbb{C}$ or $\mathbb{H}$. In the remaining cases (corresponding to projective planes) the dimension of $C$ is computed to be 5,8 , 14 or 26 . We then show that an isomorphism between projective spaces formed by extreme points gives rise to a homeomorphism between the convex bodies.

In section 4 we consider convex bodies in $\mathbb{R}^{n}$ whose face lattices determine projective planes and show that the set of extreme points in a projective line is semialgebraic in $\mathbb{R}^{n}$ and algebraic in case $n=5$. This is used to prove that in $\mathbb{R}^{5}$ all convex bodies with modular and irreducible face lattice are projectively equivalent to the set of $3 \times 3$ real symmetric positive-semidefinite matrices of trace 1 .

Finally, in section 5 , we consider convex sets in $\mathbb{R}^{n}$ whose face lattices determine abstract affine spaces, and we prove a result analogous to the main theorem.

We want to thank the anonymous referee for many suggestions to improve the exposition of the paper.

\section{Modular face lattices}

If $\mathcal{L}_{1}$ and $\mathcal{L}_{2}$ are lattices, their direct product is given by $\left(\mathcal{L}_{1} \times \mathcal{L}_{2}, \leq\right)$, where $(a, b) \leq(c, d)$ if and only if $a \leq c$ and $b \leq d$. It follows that the direct product of two lattices is modular if and only if the factors are modular. A lattice is called irreducible if it is not isomorphic to a direct product of two nontrivial lattices.

If $C_{1} \subset \mathbb{R}^{m}$ and $C_{2} \subset \mathbb{R}^{n}$ are convex bodies, define $C_{1} * C_{2} \subset \mathbb{R}^{m+n+1}$ as the convex hull of the union of a copy of $C_{1}$ and a copy of $C_{2}$ placed in such a way that their affine spans are disjoint and have no common directions. So $C_{1} * C_{2}$ is well defined up to a linear transformation: it is the convex join of $C_{1}$ and $C_{2}$ of largest possible dimension. For example $C *\{p t\}$ is a pyramid with base $C$. Let us say that a convex body $C$ is $*$-decomposable if $C=C_{1} * C_{2}$ for two convex bodies $C_{1}$ and $C_{2}$.

The following lemma is equivalent to a result proved by Barker [2] for cones.

Lemma 1. A convex body $C$ is $*$-decomposable if and only if its lattice of faces $\mathcal{L}(C)$ is reducible.

Proof. Let $C=C_{1} * C_{2}$. Observe that each point $x$ of $C_{1} * C_{2}$ with $x \notin C_{i}$ lies in a unique line segment joining a point $x_{1}$ of $C_{1}$ and a point $x_{2}$ : if $x$ lies in $x_{1} x_{2}$ and $x_{1}^{\prime} x_{2}^{\prime}$, then the lines $x_{1} x_{1}^{\prime}$ and $x_{2} x_{2}^{\prime}$ are parallel or they intersect, contradicting the assumptions on the spans of $C_{1}$ and $C_{2}$. Moreover, if $x$ moves along a straight line in $C_{1} * C_{2}$, then the corresponding points $x_{1}$ and $x_{2}$ move along straight lines in $C_{1}$ and $C_{2}$ : if $p$ and $q$ are points in $C$ and $x \in p q$, then $x=t p+(1-t) q=$ $t \lambda p_{1}+t(1-\lambda) p_{2}+(1-t) \mu q_{1}+(1-t)(1-\mu) q_{2}$, which can be rewritten as a linear combination $\alpha x_{1}+\beta x_{2}$ of the point $x_{1}=\frac{t \lambda}{t \lambda+(1-t) \mu} p_{1}+\frac{(1-t) \mu}{t \lambda+(1-t) \mu} q_{1}$ in $p_{1} q_{1}$ and the point $x_{2}=\frac{t(1-\lambda)}{t(1-\lambda)+(1-t)(1-\mu)} p_{2}+\frac{(1-t)(1-\mu)}{t(1-\lambda)+(1-t)(1-\mu)} q_{2}$ in $p_{2} q_{2}$, with $\alpha+\beta=1$, so $x$ lies in the line segment joining $x_{1}$ and $x_{2}$.

Now if $C_{i}^{\prime}$ is a face of $C_{i}$, then $C_{1}^{\prime} * C_{2}^{\prime}$ is a face of $C_{1} * C_{2}$. For, if $x \in C_{1}^{\prime} * C_{2}^{\prime}$ and $x=\lambda p+(1-\lambda) q$ with $p, q \in C_{1} * C_{2}$, then $x_{1}$ lies in $p_{1} q_{1}$ and $x_{2}$ lies in $p_{2} q_{2}$ so as $C_{i}^{\prime}$ is a face of $C_{i}, p_{i}$ and $q_{i}$ lie in $C_{i}^{\prime}$; therefore $p$ and $q$ lie in $C_{1}^{\prime} * C_{2}^{\prime}$. Conversely, if $C^{\prime}$ is a face of $C_{1} * C_{2}$ and $p \in C^{\prime}$, then $p_{1}$ and $p_{2}$ lie in $C^{\prime}$, so $C^{\prime}=\left(C^{\prime} \cap C_{1}\right) *\left(C^{\prime} \cap C_{2}\right)$. It remains to show that $C^{\prime} \cap C_{i}$ is a face of $C_{i}$. If $x \in C^{\prime} \cap C_{1}$ and $x=\lambda p+(1-\lambda) q$ 
with $p, q \in C_{1} * C_{2}$, then as $C^{\prime}$ and $C_{1}=C_{1} * \emptyset$ are faces of $C_{1} * C_{2}, p$ and $q$ lie in $C^{\prime}$ and also in $C_{1}$, so $C^{\prime} \cap C_{1}$ is a face of $C_{1}$. Similarly $C^{\prime} \cap C_{2}$ is a face of $C_{2}$. So $\mathcal{L}\left(C_{1} * C_{2}\right) \simeq \mathcal{L}\left(C_{1}\right) \times \mathcal{L}\left(C_{2}\right)$.

If $\mathcal{L}(C) \approx \mathcal{L}_{1} \times \mathcal{L}_{2}$, then $\mathcal{L}_{1}$ and $\mathcal{L}_{2}$ are isomorphic to sublattices of $\mathcal{L}(C)$, so $\mathcal{L}_{i} \approx \mathcal{L}\left(C_{i}\right)$ for two faces of $C$ with $C_{1} \wedge C_{2}=\varnothing$ and $C_{1} \vee C_{2}=C$. To show that $C=C_{1} * C_{2}$ we need to prove that $\operatorname{span}\left(C_{1}\right)$ and $\operatorname{span}\left(C_{2}\right)$ are disjoint and have no directions in common. Suppose that $x \in \operatorname{span}\left(C_{1}\right) \cap \operatorname{span}\left(C_{2}\right)$. Take $x_{i} \in \operatorname{Int}\left(C_{i}\right)$. Then the line through $x$ and $x_{i}$ meets $\partial C_{i}$ at two points $a_{i}$ and $b_{i}$. As $a_{2}$ lies in a proper subface $C_{2}^{\prime}$ of $C_{2}$, the face generated by $C_{1}$ and $a_{2}$ lies in $C_{1} \vee C_{2}^{\prime}$, which is a proper subface of $C_{1} \vee C_{2}$. But the points $a_{1}, b_{1}, a_{2}, b_{2}$ determine a plane quadrilateral whose side $a_{i} b_{i}$ lies in the interior of $C_{i}$, so its diagonals intersect at an interior point $c$ of $C_{1} \vee C_{2}$, so the face generated by $C_{1}$ and $a_{2}$ (which contains c) must be $C_{1} \vee C_{2}$, a contradiction. Now suppose that $\operatorname{span}\left(C_{1}\right)$ and $\operatorname{span}\left(C_{2}\right)$ have a common direction $v$. Take $x_{i} \in \operatorname{Int}\left(C_{i}\right)$. Then the line through $x_{i}$ in the direction $v$ meets $\partial C_{i}$ at two points $a_{i}$ and $b$. As before $a_{1}, b_{1}, a_{2}, b_{2}$ determine a plane quadrilateral whose diagonals intersect at an interior point $c$ of $C_{1} \vee C_{2}$, but $c$ lies in the face generated by $C_{1}$ and $a_{2}$, which is a proper face of $C_{1} \vee C_{2}$.

We recall now the relation between modular, irreducible lattices and projective spaces. A projective space consists of a set $P$ (the points) and a set $L$ (the lines) so that

(1) There are at least two lines; each line contains more than 2 points.

(2) Each pair of points is contained in a unique line.

(3) If $a, b, c, d$ are distinct points and the lines $a b$ and $c d$ intersect, so do the lines $a c$ and $b d$.

One also requires that all chains of subspaces (points, lines and projective spaces) ordered by inclusion have finite length. The maximum length of a chain starting with a point and ending with the projective space is its rank. The classic examples of projective spaces of rank $n$ are provided by the set of linear subspaces of a vector space $\mathbb{K}^{n+1}$, with points corresponding to the 1-dimensional subspaces and lines to the 2-dimensional subspaces. Two projective spaces are isomorphic if there is a bijective map between their sets of points that preserves the lines.

It follows from the axioms that the lattice of subspaces of a projective space is atomic, algebraic, modular and irreducible. Conversely, any lattice with these properties is the lattice of subspaces of a projective space whose points are the atoms, and the lines are the joins of 2 atoms [6]. It is a classic result of Hilbert [9] that a projective space in which Desargues theorem holds is isomorphic to the projective space $\mathbb{A P}^{n}$ determined by the linear subspaces of $\mathbb{A}^{n+1}$, for some division ring $\mathbb{A}$, and that $\mathbb{A P}^{n}$ and $\mathbb{B P}^{m}$ are isomorphic if and only if $\mathbb{A}$ and $\mathbb{B}$ are isomorphic and $m=n$. All projective spaces of rank larger than 2 are Desarguesian, but there are many non-Desarguesian projective planes.

Examples of convex bodies whose face lattices determine the projective spaces $\mathbb{R} \mathbb{P}^{n}, \mathbb{C P}^{n}, \mathbb{H P}^{n}$ and an octonionic projective plane arise from linear algebra, as cross-sections of some cones of matrices:

Lemma 2. The set $C_{n}(\mathbb{F})$ of $n \times n$ positive-semidefinite Hermitian matrices with coefficients in $\mathbb{F} \in\{\mathbb{R}, \mathbb{C}, \mathbb{H}\}$ is a real cone whose face lattice is isomorphic to the lattice of linear subspaces of $\mathbb{F}^{n}$. Each cross-section of $C_{n}(\mathbb{F})$ is a convex body 
whose set of extreme points is homeomorphic to $\mathbb{F P}^{n-1}$ by a homeomorphism that identifies faces with subspaces.

Proof. Recall that $A$ is Hermitian if $\bar{A}=A^{T}$ and is positive-semidefinite if $\bar{v} A v^{T} \geq 0$ for all $v \in \mathbb{F}^{n}$. Consider the function from $C_{n}(\mathbb{F})$ to the set of subspaces of $\mathbb{F}^{n}$ that sends each matrix $A$ to the orthogonal complement of its kernel, $(\operatorname{ker} A)^{\perp}$. This function is certainly surjective and not injective; however, for matrices of rank 1 only the scalar multiples of the same matrix have the same kernel, so there is a natural continuous bijection between matrices of rank 1 in a cross section of $C_{n}(\mathbb{F})$ and 1-dimensional linear subspaces of $\mathbb{F}^{n}$.

We claim that the matrices in $C_{n}(\mathbb{F})$ are arranged in the faces of the cone according to their kernels, with invertible matrices lying in the interior of the cone and matrices of minimal rank lying in minimal faces. To see this, let $A, B \in C_{n}(\mathbb{K})$, and let $F(B)$ be the face generated by $B$. Then

$A \in F(B) \Longleftrightarrow$

$\exists \lambda>0$ such that $B-\lambda A \in C_{n}(\mathbb{K}) \Longleftrightarrow$

$\exists \lambda>0$ such that $\bar{w} B w^{T} \geq \lambda \bar{w} A w^{T} \geq 0$ for all $w \in \mathbb{F}^{n} \Longleftrightarrow$

$\bar{w} B w^{T}=0$ implies $\bar{w} A w^{T}=0$ for all $w \in \mathbb{F}^{n} \Longleftrightarrow$

$\operatorname{ker} A \supseteq \operatorname{ker} B$, since for $A \in C_{n}(\mathbb{K}), \bar{w} A w^{T}=0$ if and only if $A w^{T}=0$.

Therefore $F(A) \rightarrow(\operatorname{ker} A)^{\perp}$ defines a bijection $\varphi$ from the set of faces of $C_{n}(\mathbb{K})$ to the set of linear subspaces of $\mathbb{K}^{n}$. To prove that $\varphi$ is an isomorphism of lattices observe that $\varphi(F(A) \vee F(B))=\varphi(F(A+B))=(\operatorname{ker}(A+B))^{\perp}=(\operatorname{ker} A \cap \operatorname{ker} B)^{\perp}=$ $\left.(\operatorname{ker} A)^{\perp} \vee(\operatorname{ker} B)^{\perp}\right)=\varphi F(A) \vee \varphi F(B)$, and on the other hand, if $F(A) \wedge F(B)$ is a nonempty face, then it is generated by a matrix $C$ with $\operatorname{ker} C=\operatorname{ker} A \vee \operatorname{ker} B$, so $\varphi(F(A) \wedge F(B))=(\operatorname{ker} C)^{\perp}=(\operatorname{ker} A)^{\perp} \cap(\operatorname{ker} B)^{\perp}=\varphi F(A) \wedge \varphi F(B)$.

Lemma 3. Let $H_{3}(\mathbb{O})$ be the set of $3 \times 3$ Hermitian matrices over the octonions. Then the subset $C_{3}(\mathbb{O})$ consisting of sums of squares of elements in $H_{3}(\mathbb{O})$ is a real cone whose face lattice determines an octonionic projective plane.

Proof. We use the nontrivial fact that each matrix in $H_{3}(\mathbb{O})$ is diagonalizable by an automorphism of $H_{3}(\mathbb{O})$ that leaves the trace invariant; see [1]. Then:

(a) A matrix $A$ in $H_{3}(\mathbb{O})$ lies in $C_{3}(\mathbb{O})$ if and only if it can be diagonalized to a matrix $A^{\prime}$ with nonnegative entries, because if $A$ lies in $C_{3}(\mathbb{O})$, then $A^{\prime}$ is a sum of squares of matrices in $H_{3}(\mathbb{O})$, which have nonnegative diagonal entries.

(b) All the idempotent matrices in $H_{3}(\mathbb{O})$ lie in $C_{3}(\mathbb{O})$ as they are squares $(A=$ $\left.A^{2}\right)$. The idempotent matrices of trace 1 correspond to the extreme rays of $C_{3}(\mathbb{O})$ since they cannot be written as nonnegative combinations of other idempotent matrices.

(c) Each face of $C_{3}(\mathbb{O})$ is generated by an idempotent matrix, because in any cone all the positive linear combinations of the same set of vectors generate the same face, so a diagonal matrix with nonnegative entries generates the same face as a matrix with only zeros and ones.

(d) Any two idempotent matrices of trace 1 lie in a face generated by an idempotent matrix of trace 2, because they can be put simultaneously in the form $\left[\begin{array}{ccc}a & x & 0 \\ x & b & 0 \\ 0 & 0 & 0\end{array}\right]$, and these lie in the face generated by $\left[\begin{array}{lll}1 & 0 & 0 \\ 0 & 1 & 0 \\ 0 & 0 & 0\end{array}\right]$.

(e) $A \in C_{3}(\mathbb{O})$ is an idempotent of trace 1 if and only if $I-A$ is an idempotent with trace 2. If $A$ and $B$ are idempotents of trace 1 , then $A$ lies in the face generated by $I-B$ if and only if $B$ lies in the face generated by $I-A$. This duality and (d) 
show that any two faces generated by idempotent matrices of trace 2 meet in a face generated by an idempotent matrix of trace 1 .

\section{Face lattices Defining PROJective spaces}

If $C$ is a convex body in $\mathbb{R}^{n}$ whose face lattice is modular and irreducible and $C$ is not strictly convex, the extreme points of $C$ form a projective space with subspaces determined by the faces of $C$. The main result of the paper is the following:

Theorem 1. Let $C$ be a convex body whose face lattice defines a projective space of rank $r$. Then:

If $r=2, C$ has dimension 5, 8, 14 or 26 .

If $r>2$ or the projective space is Desarguesian, there is a face-preserving homeomorphism from $C$ to a cross-section of a cone of positive-semidefinite Hermitian matrices over $\mathbb{R}, \mathbb{C}$, or $\mathbb{H}$.

In order to prove the theorem, we need to show that among the infinitely many isomorphism classes of projective spaces of each rank, those defined by the extreme points of convex bodies are of a very special kind, namely, topological projective spaces which are compact and connected. A topological projective space is a projective space in which the sets of subspaces of each rank are given nontrivial topologies that make the join and meet operations $\vee$ and $\wedge$ continuous, when restricted to pairs of subspaces of fixed ranks whose join or meet have a fixed rank [8].

A natural topology for the set of faces of a convex body is given by the Hausdorff metric on $\mathbb{R}^{n}$. By the Blaschke selection theorem [7], the space of all compact, convex subsets of a convex body in $\mathbb{R}^{n}$, with the Hausdorff metric, is compact. The space of faces of a convex body, however, is not compact in general.

Lemma 4. If $C$ is a convex body whose face lattice $\mathcal{F}(C)$ is modular, then the set $\mathcal{F}_{h}(C)$ of faces of rank $h$ is compact for each $h$.

Proof. Let $F_{i}$ be a sequence of faces of rank $h$. By Blaschke's theorem, $F_{i}$ has a subsequence $F_{i_{j}}$ that converges to a convex set $K$ contained in $\partial C$, so $K$ generates a proper face $F$. We claim that the rank of $F$ is $h$ and that $K$ is all of $F$.

If the rank of $F$ was less than $h$, there would be a face $F^{c}$ of $C$ of rank $n-h$ with $F^{c} \cap F=\phi$. As $F$ and $F^{c}$ are two disjoint compact sets in $\mathbb{R}^{n}$, there exists $\epsilon>0$ such that the $\varepsilon$-neighborhoods of $F$ and $F^{c}$ in $\mathbb{R}^{n}$ are disjoint. But as $F_{i_{j}} \rightarrow K \subset F$ in the Hausdorff metric, then for sufficiently large $j, F_{i_{j}}$ is contained in the $\varepsilon$-neighborhood of $F$; therefore $F_{i_{j}} \cap F^{c}=\varnothing$, but these 2 faces have ranks that add up to $n$, so they should meet, a contradiction.

If the rank of $F$ is $h$ and $K \neq F$, there is an extreme point $p \in F-K$. Let $F^{\prime}$ be a face of rank $n-h$ that meets $F$ only at $p\left(F^{\prime}=F^{c} \vee p\right.$, where $F^{c}$ is a complementary face of $F$ ) so $F^{\prime} \cap K=\varnothing$ and the previous argument gives a contradiction.

To show that the rank of $F$ cannot be larger than $h$, proceed inductively on $n-h$. As a limit of proper faces is contained in a proper face, the claim holds if $h=n-1$. Given a sequence $F_{i}$ of faces of rank $h$, let $F$ be a face generated by the limit of a convergent subsequence $F_{i_{j}}$. If $p$ is an extreme point of $C$ not in $F$, then for sufficiently large $j, p \notin F_{i_{j}}$ (otherwise $p$ would be in $F$ ). Let $G_{i_{j}}$ be a face of rank $h+1$ containing $F_{i_{j}}$ and $p$. By induction we can assume that the limit of a convergent subsequence of $G_{i_{j}}$ generates a face $G$ of rank $h+1$. Then $G$ contains $F$ properly (because it contains $p$ ), so the rank of $F$ is smaller than $h+1$. 
Lemma 5. If $C$ is a convex body whose face lattice is modular and irreducible, then $C$ is strictly convex or the set of extreme points $\mathcal{E}(C)$ is a topological projective space which is compact and connected.

Proof. The points of the projective space are the extreme points of $C$, and the points of a projective subspace are the extreme points of a face of $C$, so the projective lines are the boundaries of the strictly convex faces of $C$. With the natural topology for the set of subspaces given by the Hausdorff distance between the faces, the set of points $\mathcal{E}(\mathcal{C})=\mathcal{F}_{0}(C)$ is compact (Lemma 4) and each line is a topological sphere of dimension at least 1 . As every pair of points in $\mathcal{E}(C)$ is contained in one of these spheres, $\mathcal{E}(C)$ is connected.

It remains to show that $\vee$ and $\wedge$ are continuous on the preimages of each $\mathcal{F}_{h}(C)$. Suppose $A_{i} \rightarrow A, B_{i} \rightarrow B$, where all $A_{i} \wedge B_{i}$ and $A \wedge B$ are faces of the same rank $h$. We need to show that $A_{i} \wedge B_{i} \rightarrow A \wedge B$. By Lemma 4, $C_{i}=A_{i} \wedge B_{i}$ has convergent subsequences and the limit of a convergent subsequence $C_{i_{\alpha}}$ is a face $C_{\alpha}$ of rank $h$. As $C_{i_{\alpha}}$ is contained in $A_{i_{\alpha}}$ and $B_{i_{\alpha}}, C_{\alpha}$ is contained in $A \wedge B$. But $C_{\alpha}$ and $A \wedge B$ are both faces of rank $h$, so $C_{\alpha}=A \wedge B$. Similarly, if $A_{i} \rightarrow A, B_{i} \rightarrow B$ and $A_{i} \vee B_{i}, A \vee B$ are faces of rank $h$, the limit of each convergent subsequence of $D_{i}=A_{i} \vee B_{i}$ is a face $D$ of rank $h$. As $D_{i}$ contains $A_{i}$ and $B_{i}, D$ contains $A \vee B$, and as both faces have rank $h$ they must be equal.

Proof of Theorem 1. Assume first that the projective space determined by $\mathcal{E}(C)$ is Desarguesian. It is well known that every Desarguesian topological projective space of rank $r$ is isomorphic to the space of linear subspaces of a vector space $A^{r+1}$ for some topological division ring $A$, and this isomorphism is a homeomorphism [8, p. 1261]. A can be identified with a projective line minus a point, and in our case the projective lines are topological spheres. By a classic result of Pontryagin 8 , p. 1263] the only locally compact, connected division rings are $\mathbb{R}, \mathbb{C}$ and $\mathbb{H}$, so the topological projective space determined by $\mathcal{E}(C)$ must be isomorphic to $\mathbb{R P}^{r}, \mathbb{C P}^{r}$ or $\mathbb{H} \mathbb{P}^{r}$. The cones of Hermitian matrices described in Lemma 2 also determine these topological projective spaces, so for some cone section $C^{\prime}$, there exists a homeomorphism from $\mathcal{E}(C)$ to $\mathcal{E}\left(C^{\prime}\right)$ that is face-preserving. The following lemma shows that this homeomorphism can be extended to a face-preserving homeomorphism from $C$ to $C^{\prime}$ :

Lemma 6. If $C$ and $C^{\prime}$ are convex bodies with $\mathcal{F}(C)$ and $\mathcal{F}\left(C^{\prime}\right)$ compact, then any continuous "face-preserving" map $\varphi: \mathcal{E}(C) \rightarrow \mathcal{E}\left(C^{\prime}\right)$ extends naturally to a continuous map $\varphi: C \rightarrow C^{\prime}$.

We defer the proof of Lemma 6 to consider the remaining case of Theorem 1. Non-Desarguesian projective planes are not classified, but all the lines of a topological projective plane $\mathcal{P}$ are homeomorphic because if $l$ is a line and $p$ is a point not in $l$, then the projection $\phi: \mathcal{P}-p \rightarrow l, \phi(x)=(x \vee p) \wedge l$ is continuous and its restriction to each projective line not containing $p$ is one-to-one. When the projective lines are topological spheres, as in our case, a famous result of Adams [8, p. 1278] shows that their dimension must be $d=0,1,2,4$ or 8 .

To compute the dimension of $C$ take 3 faces of rank $1, F_{0}, F_{1}$ and $F_{2}$ so that $F_{1}$ and $F_{2}$ meet at a point $p$ not in $F_{0}$. The proof of Lemma 6 shows that the projection $\phi: \mathcal{E}(C)-\{p\} \rightarrow \partial F_{0}$ extends to a continuous map $\phi$ from the union of the faces of rank 1 of $C$ that do not contain $p$ to $F_{0}$, whose restriction to each face is one-to-one. The union of the interiors of these faces is an open subset 
$U$ of $\partial C$ and the function $\Phi: U \rightarrow F_{0} \times\left(\partial F_{1}-\{p\}\right) \times\left(\partial F_{2}-\{p\}\right)$ defined as $\Phi(x)=\left(\phi(x), \partial F(x) \wedge \partial F_{1}, \partial F(x) \wedge \partial F_{2}\right)$ is continuous and bijective, so $U$ has the same dimension as $F \times \partial F \times \partial F$, which is $3 d+1$; therefore $C$ has dimension $3 d+2$. Note that the discrepancy between the dimensions of the union of the boundaries of the faces $(2 d)$ and the union of the faces $(3 d+1)$ arises because the boundaries of the faces overlap (as the lines in a projective plane do) but the interiors of the faces are disjoint. When $r>2$, there is a similar homeomorphism from an open subset of $\partial C$ and a product $F_{0} \times\left(\partial F_{1}-\{p\}\right) \times\left(\partial F_{2}-\{p\}\right) \times \ldots \times\left(\partial F_{r}-\{p\}\right)$, where $F_{0}$ is a face of rank $r-1$ and $F_{1}, F_{2}, \ldots, F_{r}$ are faces of rank 1 . So $\operatorname{dim}(C)=\operatorname{dim}\left(F_{0}\right)+r d+1$, and it follows by induction that $\operatorname{dim} C=\frac{r(r-1)}{2} d+r-1$.

The proof of Lemma 6 relies on the following (probably known) result:

Lemma 7. Let $\mathcal{C}$ be a convex body and $\mathcal{B}(C) \subset C$ be the set of baricenters of faces of $C$. If $\mathcal{F}(C)$ is compact, then the function $b: \mathcal{F}(C) \rightarrow \mathcal{B}(C)$ that maps each face to its baricenter is a homeomorphism. Moreover, a sequence of faces $F_{i}$ converges to a face $F$ if and only if $\mathcal{E}\left(F_{i}\right)$ converges to $\mathcal{E}(F)$.

Proof. The function that assigns to each compact convex set in $\mathbb{R}^{n}$ its baricenter is continuous, so $b: \mathcal{F}(C) \rightarrow \mathcal{B}(C)$ is a continuous bijective map from a compact space to a Hausdorff space, so it is also a closed map. This proves the first part.

To prove the second part observe that the Hausdorff distance between two compact convex sets is bounded above by the Hausdorff distance between their sets of extreme points.

If $F_{i}$ converges to $F$ but $\mathcal{E}\left(F_{i}\right)$ does not converge to $\mathcal{E}(F)$, then there is a subsequence $\mathcal{E}\left(F_{i_{j}}\right)$ that stays at a distance at least $\varepsilon>0$ from $\mathcal{E}(F)$. For each $i_{j}$ there is an extreme point $p_{i_{j}} \in F_{i_{j}}$ whose distance from $\mathcal{E}(F)$ is larger than $\varepsilon$, or an extreme point $q_{i} \in F$ whose distance from $\mathcal{E}\left(F_{i_{j}}\right)$ is larger than $\varepsilon$. If there is a convergent subsequence $p_{i_{k}} \rightarrow p \in F$, then $p$ is at a distance at least $\varepsilon$ from $\mathcal{E}(F)$, so $p$ can't be an extreme point of $C$.

If there is a convergent subsequence $q_{i_{k}} \rightarrow q \in F$, take $p_{i_{k}}^{\prime} \in F_{i_{k}}$ with $p_{i_{k}}^{\prime} \rightarrow q$. Eventually $\left|p_{i_{k}}^{\prime}-q_{i_{k}}\right|<\frac{\varepsilon}{2}$, so the distance from $p_{i_{k}}^{\prime}$ to $\mathcal{E}\left(F_{i_{k}}\right)$ is at least $\frac{\varepsilon}{2}$, so $p_{i_{k}}^{\prime}$ is the center of a straight interval $I_{i_{k}}$ of length $\varepsilon$ contained in $F_{i_{k}}$. A convergent subsequence of these intervals yields a straight interval centered at $q$ and contained in $F$, so $q$ can't be an extreme point of $C$, contradicting the compacity of $\mathcal{E}(C)$.

Proof of Lemma 6. The map $\varphi: \mathcal{E}(C) \rightarrow \mathcal{E}\left(C^{\prime}\right)$ determines a function $\Psi: \mathcal{F}(C) \rightarrow$ $\mathcal{F}\left(C^{\prime}\right) . \Psi$ is continuous because by Lemma $7 F_{i} \rightarrow F$ implies $\mathcal{E}\left(F_{i}\right) \rightarrow \mathcal{E}(F)$. Then uniform continuity of $\varphi$ on $\mathcal{E}(C)$ implies that $\varphi\left(\mathcal{E}\left(F_{i}\right)\right) \rightarrow \varphi(\mathcal{E}(F)$ ), so by definition $\mathcal{E}\left(\Psi\left(F_{i}\right)\right) \rightarrow \mathcal{E}(\Psi(F))$ and so $\Psi\left(F_{i}\right) \rightarrow \Psi(F)$. So $\varphi$ can be extended to a continuous function $\varphi: \mathcal{B}(C) \rightarrow \mathcal{B}\left(C^{\prime}\right)$ as $b \circ \Psi \circ b^{-1}$ (recall that $\mathcal{E}(C) \subset \mathcal{B}(C)$ ). Now we can extend $\varphi$ to the interiors of the faces of $C$, starting with the faces of smallest dimension and using the function already defined on the boundaries of faces and their baricenters to extend it linearly on rays to their interiors. The delicate point is to prove that the extension is continuous, as $C$ may have an infinite number of faces.

For each point $a \in C$, let $F(a)$ be the face of $C$ generated by $a$ and let $b(a)$ be the baricenter of $F(a)$. Although $F(a)$ and $b(a)$ are not continuous functions of $a$ on all of $C$, they are continuous on the union of the interiors of the faces of dimension $d$, for each $d$. To see this suppose $a_{i} \rightarrow a$ is a converging sequence of points lying 
in the interiors of faces $F_{i}$ and $F$ of dimension $d$. As $\mathcal{F}(C)$ is compact, $F_{i}$ has a subsequence converging to a face $F^{\prime}$ of dimension at most $d$. As $a$ is contained in $F^{\prime}$ and $a$ is contained in the interior of $F, F$ is a face of $F^{\prime}$. As the dimension of $F^{\prime}$ is at most that of $F$, so $F=F^{\prime}$. This shows that $F(a)$ is continuous. The continuity of $b(a)$ follows.

Now if $a \neq b(a)$ let $p(a)$ be the projection of $a$ to $\partial F(a)$ from $b(a)$ and let $\lambda(a)=\frac{|a-b(a)|}{|p(a)-b(a)|}$ (or 0 if $\left.a=b(a)\right)$ so $a=(1-\lambda(a)) b(a)+\lambda(a) p(a)$. Define $\varphi(a)=(1-\lambda(a)) \varphi(b(a))+\lambda(a) \varphi(p(a))$.

Assume inductively that $\varphi$ is continuous on the closed set formed by the union of $\mathcal{B}(C)$ and the faces of $C$ of dimension less than $d$, and let us show that for each sequence of points $a_{i}$ in the interiors of faces of dimension $d, a_{i} \rightarrow a$ implies $\varphi\left(a_{i}\right) \rightarrow \varphi(a)$. We may assume that the $a_{i}$ are not baricenters, so $p\left(a_{i}\right)$ is well defined.

Case 1. If $F\left(a_{i}\right) \rightarrow F(a)$, then $b\left(a_{i}\right) \rightarrow b(a)$ by the continuity of $b$ on faces.

If $b(a) \neq a$, then $p\left(a_{i}\right) \rightarrow p(a)$ and $\lambda\left(a_{i}\right) \rightarrow \lambda(a)$, so $\varphi\left(a_{i}\right)=\left(1-\lambda\left(a_{i}\right)\right) \varphi\left(b\left(a_{i}\right)\right)+$ $\lambda\left(a_{i}\right) \varphi\left(p\left(a_{i}\right)\right) \rightarrow(1-\lambda(a)) \varphi(b(a))+\lambda(a) \varphi(p(a))=\varphi(a)$.

If $b(a)=a$, then $\lim b\left(a_{i}\right)=\lim a_{i}$, but $p\left(a_{i}\right)$ may not converge, so consider a convergent subsequence $p\left(a_{i_{j}}\right):$ If $\lim p\left(a_{i_{j}}\right) \neq \lim b\left(a_{i_{j}}\right)=\lim a_{i_{j}}$, then $\lim \lambda\left(a_{i_{j}}\right)=$ 0 , so $\varphi\left(a_{i_{j}}\right)=\varphi\left(b\left(a_{i_{j}}\right)\right)+\lambda\left(a_{i_{j}}\right)\left[\varphi\left(p\left(a_{i_{j}}\right)\right)-\varphi\left(b\left(a_{i_{j}}\right)\right)\right] \rightarrow \varphi(b(a))+0=\varphi(a)$. If $\lim p\left(a_{i_{j}}\right)=\lim b\left(a_{i_{j}}\right)$, then $\lim \varphi\left(p\left(a_{i_{j}}\right)\right)=\lim \varphi\left(b\left(a_{i_{j}}\right)\right.$ ) (by continuity of $\varphi$ in the baricenters and faces of lower dimension) and as $\varphi\left(a_{i_{j}}\right)$ lies between them, $\lim \varphi\left(a_{i_{j}}\right)=\lim \varphi\left(b \circ F\left(a_{i_{j}}\right)\right)=\varphi(b(a))=\varphi(a)$.

Case 2. If $F\left(a_{i}\right) \nrightarrow F(a)$, then for any convergent subsequence $F\left(a_{i_{j}}\right)$ with limit a face $F \neq F(a), a$ lies in $F$ and so $a$ must lie in $\partial F$, so $\left|a_{i_{j}}-p\left(a_{i_{j}}\right)\right| \rightarrow 0$ and $\lambda\left(a_{i_{j}}\right) \rightarrow 1$, so $\lim \varphi\left(a_{i_{j}}\right)=\lim \left(1-\lambda\left(a_{i_{j}}\right)\right) \varphi\left(b\left(a_{i_{j}}\right)\right)+\lambda\left(a_{i_{j}}\right) \varphi\left(p\left(a_{i_{j}}\right)\right)=$ $\lim \varphi\left(p\left(a_{i_{j}}\right)\right)=\varphi(a)$ (by continuity of $\varphi$ on the faces of lower dimension).

\section{Projective Planes AND the CASE $n=5$}

Let's now consider more closely a convex body in $\mathbb{R}^{n}$ whose face lattice determines a projective plane, i.e., a convex body $C$ different from a triangle in which every pair of extreme points lies in a face and every pair of faces with more than one point meet. By the proof of Theorem $1, \mathcal{F}_{0}(C)$ and $\mathcal{F}_{1}(C)$ are closed manifolds of dimension $2 d$ and $C$ has dimension $n=3 d+2$ for some $d \in\{1,2,4,8\}$.

Lemma 8. If the face lattice of a convex body $C$ in $\mathbb{R}^{n}$ determines a projective plane, the boundaries of the faces of rank 1 of $C$ are semi-algebraic sets. If $n=5$, they are conic sections.

The key to the proof of this lemma is the following:

Lemma 9. If $C$ is a convex body in $\mathbb{R}^{n}$ whose face lattice determines a projective plane, then each affine subspace of dimension $d+1$ of $\mathbb{R}^{n}$ that meets the affine spans of all faces in $\mathcal{F}_{1}(C)$ is the affine span of one of these faces.

Proof. In the following, span will mean affine span. Let $S$ be an affine subspace of dimension $d+1$ that intersects $\operatorname{span}(F)$ for every $F$ in $\mathcal{F}_{1}(C)$. Then the set $\left\{F \in \mathcal{F}_{1}(C) ; \operatorname{dim}(S \cap \operatorname{span}(F)) \geq i\right\}$ is closed in $\mathcal{F}_{1}(C)$, for each $i$.

Case 1. $d=1$. We claim that if $S$ is not the span of a face $F$, then $S$ cannot intersect $\operatorname{span}(F)$ in more than one point. For, if $S \cap \operatorname{span}(F)$ contains a line, then 
$\operatorname{span}(S \cup F)$ is 3-dimensional. Take an extreme point $p \notin \operatorname{span}(S \cup F)$ and let $F_{1}$ and $F_{2}$ be 2 faces containing $p$, and meeting $F$ at points $p_{1}$ and $p_{2}$ not in $S$. If $p_{1}^{\prime}$ and $p_{2}^{\prime}$ are points in $S \cap \operatorname{span}\left(F_{1}\right)$ and $S \cap \operatorname{span}\left(F_{2}\right)$ respectively, then $p, p_{i}$ and $p_{i}^{\prime}$ are not collinear (otherwise $p$ would be in $\operatorname{span}(S \cup F)$ ). So $\operatorname{span}(p \cup S \cup F) \supset \operatorname{span}\{p$, $\left.p_{i}, p_{i}^{\prime}\right\}$ contains $F, F_{1}$ and $F_{2}$ and it contains each 2-dimensional face that meets $F, F_{1}$ and $F_{2}$ at 3 noncollinear points, but every face is a limit of such faces, so $\operatorname{span}(p \cup S \cup F)$ contains all the faces of $C$. But $\operatorname{span}(p \cup S \cup F)$ has dimension 4, contradicting the fact that $C$ has dimension 5 .

This shows that $S$ intersects each $\operatorname{span}(F)$ at exactly one point, and so $S$ contains at most one extreme point $p$ of $C$. The function $I: \mathcal{F}_{1}(C) \rightarrow S$ that maps each face $F_{i}$ to the point of intersection of $\operatorname{span}(F)$ with $S$ is continuous, and as the spans of faces meet only at extreme points, $I$ is injective outside the set of faces $\mathcal{F}_{1}^{p}(C)$ containing the extreme point $p$ in $S$. As $d=1, \mathcal{F}_{1}(C)$ is topologically a closed surface and $\mathcal{F}_{1}^{p}(C)$ is a closed curve, but there are no continuous maps from a closed surface to a plane that fail to be injective only along a curve.

Case 2. $S$ does not contain extreme points of some face $F$ in $\mathcal{F}_{1}(C)$. Choose $F$ to minimize the dimension of the affine subspace $S \cap \operatorname{span}(F)$ of $S$. Then for every face $F^{\prime}$ in a neighborhood of $F, S \cap \operatorname{span}\left(F^{\prime}\right)$ is an affine subspace of minimal dimension of $S$ that has no extreme points of $F^{\prime}$. If $S^{0}$ is a maximal subspace of $S$ meeting $\operatorname{span}(F)$ in one point, then $S^{0}$ meets $\operatorname{span}\left(F^{\prime}\right)$ in one point for all $F^{\prime}$ in a smaller neighborhood $V$ of $F$. Then the function $I: V \rightarrow S^{0}$ that maps $F^{\prime}$ to $S^{0} \cap \operatorname{span}\left(F^{\prime}\right)$ is continuous, and it is injective as the spans of faces only meet at extreme points. But an injective map between manifolds can only exist when the domain has dimension no larger than the target, so $2 d=\operatorname{dim} V \leq \operatorname{dim} S^{0} \leq \operatorname{dim} S \leq d+1$, so $d=1$ and we are in case 1.

Case 3. $S$ contains extreme points of each face $F$. If $S$ intersects $C$ only in its boundary, then $S \cap C$ is contained in a face $F_{1}$ of $C$ and so either $S \cap C=F_{1}$ (therefore $\operatorname{span}\left(F_{1}\right)=S$ ) or there is an extreme point $p$ of $F_{1}$ not contained in $S$, but then a face $F_{2}$ that meets $F_{1}$ at $p$ does not meet $S \cap F_{1}=S \cap C$ and so $S$ does not contain extreme points of $F_{2}$, a contradiction.

If $S$ meets the interior of $C$, then $S \cap \partial C=\partial_{S}(S \cap C)$. Consider the set $\mathcal{F}_{1}^{p}(C)$ of faces of rank 1 containing an extreme point $p$ not in $S$. Choose $F$ in $\mathcal{F}_{1}^{p}(C)$ so that $S \cap F$ has minimal dimension. Then for all $F^{\prime}$ in some neighborhood $V$ of $F$ in $\mathcal{F}_{1}^{p}(C), S \cap F^{\prime}$ has the same dimension and the map $I_{V}: V \rightarrow S \cap \partial C$ that sends $F^{\prime}$ to the baricenter of $S \cap F^{\prime}$ is continuous and injective. As $I_{V}$ is a map between manifolds, $d=\operatorname{dim} \mathcal{F}_{1}^{p}(C)=\operatorname{dim} V \leq \operatorname{dim}(S \cap \partial C) \leq d$ and so by invariance of domain the image of $I_{V}$ is an open subset of $S \cap \partial C=\partial_{S}(S \cap C)$. As the baricenters of the faces of a convex body can fill an open set of its boundary only if the open set consists of extreme points, the image of $I_{V}$ consists of extreme points of $S \cap C$ that (by the assumption that $S$ contains extreme points of each $F^{\prime}$ in $V$ ) must be extreme points of $C$. So the line segment joining two extreme points in the image of $I_{V}$ lies in $\operatorname{Int}_{S}(S \cap C)$, and at the same time it lies in a face of $C$, so it must lie in $S \cap \partial C=\partial_{S}(S \cap C)$, a contradiction.

Proof of Lemma 8 . By the previous lemma, the set $\mathcal{S}$ of spans of faces of $C$ is the same as the set of $(d+1)$-dimensional affine subspaces of $\mathbb{R}^{n}$ that intersect every element of $\mathcal{S}$. The set of all $(d+1)$-dimensional affine subspaces of $\mathbb{R}^{n}$ forms a real algebraic variety, and the condition that the subspaces meet a fixed subspace 


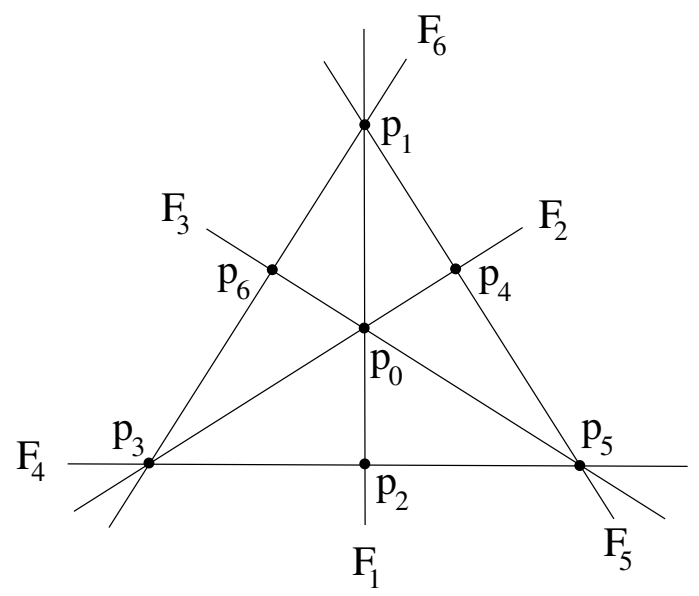

FiguRE 1

is algebraic, so (by the finite descending chain condition) there is a finite family of spans $S_{1}, S_{2}, \ldots, S_{m} \in \mathcal{S}$ such that $S \in \mathcal{S}$ if it intersects these $S_{i}$ 's (see [5]).

Now for $\left(x_{1}, x_{2}, \ldots, x_{m}\right) \in S_{1} \times S_{2} \times \cdots \times S_{m}$, the subspace $\operatorname{span}\left(x_{1}, \ldots, x_{m}\right)$ has dimension at least $d+1$ (otherwise it would be contained in two subspaces of dimension $d+1$ that meet each $S_{i}$, so they would both be in $\mathcal{S}$, but two spans can only meet in 1 point). So $\operatorname{span}\left(x_{1}, \ldots, x_{m}\right)$ lies in $\mathcal{S}$ if and only if its dimension is $d+1$, and this happens if and only if some determinants (given by polynomials in $x_{1}, \ldots, x_{m}$ ) vanish. Therefore the set $X=\left\{\left(x_{1}, x_{2}, \ldots, x_{m}\right) \in S_{1} \times S_{2} \times \cdots \times S_{m} \mid \operatorname{span}\left(x_{1}, \ldots, x_{m}\right) \in \mathcal{S}\right\}$ is real algebraic, as is the set $X^{p}$ formed by the elements of $X$ that contain a fixed point $p$. If $F_{1}$ is the face in $S_{1}$ and $p$ is an extreme point of $C$ outside $F_{1}$, then $\partial F_{1}$ consists of the intersections of $S_{1}$ with the elements of $\mathcal{S}$ containing $p$. So $\partial F_{1}$ is the one-to-one projection of the algebraic set $X^{p}$ to $S_{1}$, so $\partial F_{1}$ is at least semi-algebraic.

Now assume $n=5$, so $d=1$. Every projective plane contains 7 points and 6 lines so that each line contains 3 points as in Figure 1, so $C$ has 7 extreme points and 6 faces intersecting in that way. The 7 points are in general position in $\mathbb{R}^{5}$ because as each face of $C$ is spanned by 3 points, the span of any 6 of those points contains the span of 3 faces, which is all of $\mathbb{R}^{5}$. Therefore we may assume (by applying a projective transformation) that the 7 points are $p_{0}=(0,0,0,0,0), p_{1}=$ $(1,0,0,0,0), p_{2}=(0,1,0,0,0), \ldots, p_{5}=(0,0,0,0,1), p_{6}=(1,1,1,1,1)$. Let $S_{i}$ be the plane spanned by the face $F_{i}$. A plane $S$ that intersects $S_{1}, S_{2}$ and $S_{3}$ has a parametrization $(x, y, z, v, w)=r(a, b, 0,0,0)+s(0,0, c, d, 0)+t(e, e, e, e, f)$ with $r+s+t=1$. $S$ intersects $S_{4}, S_{5}$ and $S_{6}$ only if three systems of linear equations in $r, s, t$ represented by the following matrices have nontrivial solutions:

$\left|\begin{array}{ccc||ccc}a & 0 & e & b & 0 & e \\ 0 & d & e \\ b-1 & c-1 & 2 e+f-1\end{array}\right| \begin{array}{cccc}b & 0 & e-f \\ 0 & c & e \\ a-1 & d-1 & 2 e+f-1\end{array} \mid$ 
As the determinants of these matrices are linear functions on the variables $e$ and $f$, they vanish simultaneously if and only if the next matrix has determinant 0 :

$$
\operatorname{det}\left|\begin{array}{ccc}
-a c+2 a d-b d+a+d & a d & -a d \\
-a c+2 b c-b d+b+c & b c & -b c \\
-a d-b c+2 b d+b+d & a d+b c-b d-b-d & -b d
\end{array}\right|=0 .
$$

This determinant factors as the product of a linear and a quadratic function of $a$ and $b$ (with coefficients in $c$ and $d$ ). Since the boundary of the face $F_{1}$ is formed by the intersections of $S_{1}$ with the planes that meet all $S_{i}$ 's and go through a fixed point in the boundary of $F_{2}$ (this corresponds to fixing $c$ and $d$ ), the boundary of $F_{1}$ is contained in the union of a line and a conic. As the boundary of $F_{1}$ is strictly convex, it must be the conic.

Theorem 2. Every 5-dimensional convex body with modular and irreducible face lattice is projectively equivalent to the set of $3 \times 3$ real symmetric positive-semidefinite matrices of trace 1.

Proof. Let $C$ and $C^{\prime}$ be two convex bodies in $\mathbb{R}^{5} \subset \mathbb{R P}^{5}$ having modular and irreducible face lattices. The claim is that there is a projective transformation of $\mathbb{R P}^{5}$ taking $C$ to $C^{\prime}$. Take extreme points $p_{0}, p_{1}, \ldots, p_{6}$ and faces $F_{1}, \ldots, F_{6}$ of $C$ as in Figure 1. Pick an extreme point $p_{0}^{\prime}$ in $C^{\prime}$ and two faces $F_{1}^{\prime}$ and $F_{2}^{\prime}$ of $C^{\prime}$ intersecting at $p_{0}^{\prime}$. Let $S_{i}$ be the span of $F_{i}$. As the faces of $C$ and $C^{\prime}$ are conics, there are linear transformations from $S_{1}$ to $S_{1}^{\prime}$ taking $F_{1}$ to $F_{1}^{\prime}$ and from $P_{2}$ to $P_{2}^{\prime}$ taking $F_{2}$ to $F_{2}^{\prime}$. Together, they define a linear transformation $l$ from $\operatorname{span}\left(F_{1} \cup F_{2}\right)$ to $\operatorname{span}\left(F_{1}^{\prime} \cup F_{2}^{\prime}\right)$. Let $p_{i}^{\prime}=l\left(p_{i}\right)$ for $i=1, \ldots, 4$. The faces $F_{4}, F_{5}, F_{6}$ are generated by unique pairs of $p_{i}$ 's with $i \leq 4$. Let $F_{4}^{\prime}, F_{5}^{\prime}, F_{6}^{\prime}$ be the faces generated by the corresponding pairs of $p_{i}^{\prime}$ 's. Finally, let $p_{5}^{\prime}=S_{4}^{\prime} \cap S_{5}^{\prime}$, let $F_{3}^{\prime}$ be the face generated by $p_{0}^{\prime}$ and $p_{5}^{\prime}$ and let $p_{6}^{\prime}=S_{3}^{\prime} \cap S_{6}^{\prime}$. The linear transformation $l$ can be extended to a projective transformation $\rho$ in $\mathbb{R P}^{5}$ that takes $p_{5}$ to $p_{5}^{\prime}$ and $p_{6}$ to $p_{6}^{\prime}$. As $\rho$ sends each $p_{i}$ to $p_{i}^{\prime}$, it sends each $S_{i}$ to $S_{i}^{\prime}$, so it sends each plane in $\mathbb{R}^{5}$ intersecting every $S_{i}$ to a plane intersecting every $S_{i}^{\prime}$. Since by construction $\rho$ takes those planes that meet $\partial F_{1}$ and $\partial F_{2}$ to planes that meet $\partial F_{1}^{\prime}$ and $\partial F_{2}^{\prime}$, Lemma 9 implies that $\rho$ maps spans of faces of $C$ to spans of faces of $C^{\prime}$, and therefore it maps faces to faces.

\section{Face lattices Defining AFFine SPACES}

Projective spaces are closely related to affine spaces, whose axioms capture the basic properties of parallelism in $\mathbb{R}^{n}$. Every affine space $A$ can be embedded in a projective space by attaching to $A$ a point at infinity for each parallelism class of affine lines, and conversely, in any projective space $P$ the complement of a maximal projective subspace is an affine space. So it seems natural to look at convex sets whose faces meet as the subspaces of an affine space. For this, one needs to consider convex sets in $\mathbb{R}^{n}$ that are closed but not necessarily compact. Observe that if a closed convex set $C$ in $\mathbb{R}^{n}$ is noncompact, it contains a ray (half of a Euclidean line); and if $C$ contains a ray, then it contains all the parallel rays starting at points of $C$ (we say that $C$ contains an infinite direction). So if $C$ contains a line, $C$ is the product of that line and a closed convex set $C^{\prime}$ of lower dimension and the face lattices of $C$ and $C^{\prime}$ are isomorphic. So from now on we will assume that $C$ does not contain lines.

It is easy to see that a convex body with a finite number of faces cannot determine an affine space: the faces of rank $i$ would have dimension $i$, two parallel faces of 
rank 1 generate a face of rank 2 with at least 4 vertices, but the sides of a polygon do not define an affine plane. On the other hand, if $C$ is a cone in $\mathbb{R}^{n+1}$ whose face lattice determines a projective space, the intersection of $C$ with a hyperplane parallel to one that touches $C$ in a maximal face is a closed (noncompact) convex set in $\mathbb{R}^{n}$ whose face lattice determines an affine space. In particular, there are closed convex sets whose faces determine real, complex and quaternionic affine spaces and an octonionic affine plane.

We want to prove a result analogous to the main theorem for closed convex sets $\mathbb{R}^{n}$ whose face lattices determine affine spaces. For this we will show that these sets have compactifications in $\mathbb{R P}^{n}$ that are convex bodies whose face lattices define projective spaces. Think of $\mathbb{R P}^{n}$ as the result of attaching to $\mathbb{R}^{n}$ a "point at infinity" for each class of parallel lines, and also as the space of lines through the origin in $\mathbb{R}^{n+1}$. Identifying $\mathbb{R}^{n}$ with a hyperplane of $\mathbb{R}^{n+1}$ that does not contain the origin gives an embedding of $\mathbb{R}^{n}$ as a dense open subset of $\mathbb{R} \mathbb{P}^{n}$. The remaining points of $\mathbb{R}^{n}$ correspond to lines through the origin in $\mathbb{R}^{n+1}$ that do not meet the hyperplane, and these correspond to unoriented directions in $\mathbb{R}^{n}$. Different identifications of $\mathbb{R}^{n}$ with a hyperplane give different embeddings of $\mathbb{R}^{n}$ into $\mathbb{R} \mathbb{P}^{n}$. Define a set in $\mathbb{R P}^{n}$ to be convex if it is the image of a convex set in $\mathbb{R}^{n}$ under one of these embeddings. So convex sets in $\mathbb{R P}^{n}$ correspond to convex cones based at the origin of $\mathbb{R}^{n+1}$ (by definition a cone does not contain lines). If $C$ is a closed convex set in $\mathbb{R}^{n}$, its closure in $\mathbb{R P}^{n}$, denoted by $\bar{C}$, is obtained by attaching to $C$ a point at infinity for each infinite direction of $C$. If $C$ does not contain lines, the closure of the cone over $C$ in $\mathbb{R}^{n+1}$ is a cone, so $\bar{C}$ is convex in $\mathbb{R P}^{n}$ and the faces of $\bar{C}$ are the closures of the faces of $C$ in $\mathbb{R P}^{n}$ and the intersections of these faces with the set of points at infinity.

Lemma 10. If the faces of a closed convex set $C$ in $\mathbb{R}^{n}$ define an affine space, each face representing an affine line contains a unique infinite direction, which is the same for faces representing parallel lines.

Proof. We are assuming that $C$ does not contain lines, so the points of the affine space are the extreme points of $C$ and the affine lines are the boundaries of strictly convex faces of $C$ of dimension at least two.

We claim that a face $A$ representing an affine line cannot be compact. Otherwise, let $P$ be a face that contains $A$ and represents an affine plane. Let $p$ and $q$ be two extreme points of $A$ and let $q_{i}$ be a sequence of extreme points of $P$, not in $A$, that converge to $q$. If $A_{i}$ is the face generated by $p$ and $q_{i}$, then for sufficiently large $i, A_{i}$ lies in an $\varepsilon$-neighborhood $U_{\varepsilon}$ of $A$. If not, take a subsequence $A_{i_{j}}$ having extreme points $r_{j_{j}}$ lying outside $U_{\varepsilon}$. As $A$ is compact we may assume that the sequence $r_{i_{j}}$ is bounded and therefore has a subsequence converging to a point $r$ outside $U_{\varepsilon}$. As the triangles $p q_{i_{j}} r_{i_{j}}$ lie in $\partial P$, the triangle $p q r$ is contained in $\partial P$ and therefore is contained in a proper face of $P$. This is impossible because the only proper face of $P$ that contains $p$ and $q$ is $A$, and $r$ is not in $A$.

Now take a face $A^{\prime}$ in $P$ that does not meet $A$ (i.e., $A$ and $A^{\prime}$ represent parallel affine lines). As $A$ is compact and $A^{\prime}$ is closed in $\mathbb{R}^{n}$, there is an $\varepsilon$-neighborhood of $A$ that does not intersect $A^{\prime}$. By the previous argument there is a point $q_{i}$ not in $A$ so that the face $A_{i}$ generated by $p$ and $q_{i}$ is contained in the $\varepsilon$-neighborhood of $A$. So $A_{i}$ does not meet $A^{\prime}$, but $A$ was supposed to be the only face containing $p$ and disjoint from $A^{\prime}$, a contradiction This proves that a face $A$ representing an affine line is not compact, so it contains rays. 
The rays in $A$ passing through a point $p$ form a convex cone, and each ray $l_{+}$ in the boundary of this cone is the limit of a sequence of intervals $p p_{i}$ in $A$ joining $p$ with extreme points of $A$. If $q$ is an extreme point of $C$ outside $A, q$ and $A$ generate a face $P$ representing an affine plane. The sequence of intervals $q p_{i}$ lie in $\partial P$ and converge to a ray $m_{+}$parallel to $l_{+}$that contains $q$, so (as $P$ is closed) $m_{+}$ is contained in a face $A^{\prime}$ of $P$. As two faces that contain parallel rays cannot meet at a single point, $A^{\prime}$ does not meet $A$, so $A^{\prime}$ represents the affine line parallel to $A$ through $p$. This shows that two faces $A$ and $A^{\prime}$ representing parallel affine lines have the same cones of infinite directions. If the cones have more than one infinite direction, a line going across a cone for $A$ meets $A$ in a closed interval, and the same is true for a parallel line going across a cone for $A^{\prime}$.

So there are 4 extreme points $a_{1}, a_{2}$ and $a_{1}^{\prime}, a_{2}^{\prime}$ of $A$ and $A^{\prime}$ respectively that are coplanar in $\mathbb{R}^{n}$. They determine a convex quadrilateral whose diagonals meet at an interior point, but this is impossible because the diagonals are contained in faces representing different affine lines, and these can only meet at an extreme point

Theorem 3. If $C$ is a closed convex set in $\mathbb{R}^{n}$ whose faces determine an affine space, then there is a face-preserving homeomorphism from $C$ to a "parabolic section" of a cone of positive-semidefinite Hermitian matrices over $\mathbb{R}, \mathbb{C}$, or $\mathbb{H}$, or $C$ has dimension 8,14 or 26 .

Proof. Consider $C \subset \mathbb{R}^{n} \subset \mathbb{R P}^{n}$. By the previous lemma the faces of $C$ representing affine lines share an infinite direction in $\mathbb{R}^{n}$ if and only if they represent parallel affine lines. The closure of $C$ in $\mathbb{R P}^{n}$ contains one point at infinity for each infinite direction in $C$, so $\bar{C}$ contains an extreme point at infinity for each class of faces of $C$ representing parallel lines, and this corresponds precisely with the definition of the projective completion of an affine space (we are not assuming any topologies in the affine or the projective space).

Therefore $\bar{C}$ is a convex body in $\mathbb{R} \mathbb{P}^{n}$ whose face lattice determines a projective space, and we can apply Theorems 1 and 2 to show that there is a face-preserving homeomorphism $\varphi$ from $\bar{C}$ to an "elliptic" section of a cone of Hermitian matrices, or $\bar{C}$ (and therefore $C$ ) has dimension 8, 14 or 26. As $C$ is obtained from $\bar{C}$ by removing a maximal face, $\varphi$ maps $C$ to the set of points of the elliptic section lying outside a maximal face, and there is a natural bijection between these points and the points in a "parabolic section" of the cone obtained by intersecting the cone with a hyperplane parallel to one that touches the cone in the maximal face.

\section{Questions}

The results above suggest some questions:

Can two convex bodies of the same dimension define nonisomorphic projective planes (so they are not related by a face-preserving homeomorphism)? In dimensions 8 and 14 this is equivalent to asking if these projective planes are always Desarguesian. In dimension 26 there might be many nonisomorphic examples.

Are all the convex bodies whose face lattices determine classical projective spaces projectively equivalent to sections of cones of Hermitian matrices?

Are there examples of convex sets with face lattice isomorphic to the lattice of subspaces of a hyperbolic space?

What can be said about convex bodies whose face lattice is lower semimodular? 


\section{REFERENCES}

[1] Baez, J., The Octonions, Bull. Amer. Math. Soc. (N.S.) 39 (2002), 145-205. MR.1886087 (2003f:17003)

[2] Barker, G.P., Theory of cones, Linear Algebra Appl. 39 (1981), 263-291. MR625256 (83e:15022)

[3] Barker, G.P., Modular face lattices: Low-dimensional cases, Rocky Mountain Journal of Math. 11 (3) (1981), 435-439. MR722577 (84m:52019)

[4] Birkhoff, G., Lattice Theory, 3rd edition, Amer. Math. Soc. Colloq. Publ., AMS, 1967. MR0227053 (37:2638)

[5] Bochnak, J., Coste, M., Roy, M., Real Algebraic Geometry, Springer, 1998. MR.1659509 (2000a:14067)

[6] Crawley, P., Dilworth R.P., Algebraic Theory of Lattices, Prentice-Hall, Inc., 1973.

[7] Grubber, P.M., Willis, J.M., Handbook of Convex Geometry. Volume A, North-Holland, 1993.

[8] Grundhoefer, T., Loewen, R., Linear topological geometries, in Handbook of Incidence Geometry. Buildings and Foundations. North-Holland, 1995. MR.1360738 (97c:51008)

[9] Hartshorne, R., Foundations of Projective Geometry, W.A. Benjamin, Inc., 1967. MR 0222751 $(36: 5801)$

[10] Labardini-Fragoso, D., The face lattice of a finite dimensional cone (Spanish), Bachelor's Thesis, UNAM, 2004.

[11] Salzmann, H., Betten, D., Grundhoefer, T., Haehl, H., Loewen, R., Stroppel, M., Compact Projective Planes, De Gruyter Expositions in Mathematics, 1995. MR.1384300 (97b:51009)

Mathematisches Institut, Universität Bonn, D-53115 Bonn, Germany

E-mail address: labardini@math.uni-bonn.de

Instituto de Matemáticas, Universidad Nacional Autónoma de México, Cuernavaca, MÉXICO

E-mail address: max@matcuer.unam.mx

Instituto de Matemáticas, Universidad Nacional Autónoma de México, Cuernavaca, MÉXICO

E-mail address: takane@matcuer.unam.mx 